\title{
Infection risk with the use of interleukin inhibitors in hospitalized patients with COVID-19: A narrative review
}

\author{
Thoyaja Koritala', Vishwanath Pattan², Raghavendra Tirupathi ${ }^{3}$, Ali A. Rabaan ${ }^{4,5}$, \\ Abbas Al Mutair ${ }^{6,7,8}$, Saad Alhumaid ${ }^{9}$, Ramesh Adhikari10, Keerti Deepika ${ }^{11}$, \\ Nitesh Kumar Jain ${ }^{12}$, Vikas Bansal ${ }^{13}$, Aysun Tekin ${ }^{13}$, Simon $\mathrm{Zec}^{13}$, Amos Lal ${ }^{14}$, \\ Syed Anjum Khan ${ }^{12}$, Juan Pablo Domecq Garces ${ }^{15}$, Omar M. Abu Saleh ${ }^{16}$, Salim R. Surani17,18, \\ Rahul Kashyap ${ }^{19}$ \\ ${ }^{1}$ Division of Hospital Internal Medicine, Mayo Clinic Health System, Mankato, USA; \\ ${ }^{2}$ Department of Endocrinology, Wyoming Medical Center, Casper, USA; \\ ${ }^{3}$ Department of Medicine, Keystone Health, Chambersburg, USA; \\ ${ }^{4}$ Molecular Diagnostic Laboratory, Johns Hopkins Aramco Healthcare, Dhahran, Saudi Arabia; \\ ${ }^{5}$ Department of Public Health and Nutrition, University of Haripur, Haripur, Pakistan; \\ ${ }^{6}$ Research Center, Almoosa Specialist Hospital, Al-Ahsa, Saudi Arabia; \\ ${ }^{7}$ College of Nursing, Princess Norah Bint Abdulrahman University, Riyadh, Saudi Arabia; \\ ${ }^{8}$ School of Nursing, Wollongong University, Wollongong, Australia; \\ ${ }^{9}$ Administration of Pharmaceutical Care, Al-Ahsa Health Cluster, Al-Ahsa, Saudi Arabia; \\ ${ }^{10}$ Department of Hospital Medicine, Franciscan Health, Lafayette, USA; \\ ${ }^{11}$ Translational Health Disparities Science Research Program, Nemours Healthcare System for Children, \\ Wilmington, USA; \\ ${ }^{12}$ Division of Community Critical Care, Mayo Clinic Health System, Mankato, USA; \\ ${ }^{13}$ Research Fellow, Mayo Clinic College of Medicine and Science, Rochester, USA; \\ ${ }^{14} \mathrm{Critical}$ Care Internal Medicine Fellow, Mayo Clinic College of Medicine and Science, Rochester, USA; \\ ${ }^{15}$ Division of Nephrology and Hypertension, Mayo Clinic, Rochester, USA; \\ ${ }^{16}$ Division of Infectious Diseases, Mayo Clinic, Rochester, USA; \\ ${ }^{17}$ Adjunct Clinical Professor of Medicine and Pharmacy, Texas A\&M University, College Station, USA; \\ ${ }^{18}$ Research Collaborator (limited tenure), Mayo Clinic, Rochester, USA; \\ ${ }^{19}$ Department of Anesthesiology and Perioperative Medicine, Mayo Clinic, Rochester, USA
}

Article received 14 September, 2021; accepted 12 November, 2021

\section{SUMMARY}

Introduction: To date, only corticosteroids and interleukin-6 (IL-6) inhibitors have been shown to reduce mortality of hospitalized patients with COVID-19. In this literature review, we aimed to summarize infection risk of IL inhibitors, with or without the use of corticosteroids, used to treat hospitalized patients with COVID-19.

Methods: A literature search was conducted using the following evidence-based medicine reviews: Cochrane Central Register of Controlled Trials; Cochrane Database of Systematic Reviews; Embase; Ovid Medline; and Epub Ahead of Print, In-Process, In-Data-Review \& Other Non-Indexed Citations, Daily and Versions 1946 to April 28, 2021. All relevant articles were identi- fied using the search terms COVID-19 or SARS-coronavirus-2, infections, interleukins, inpatients, adults, and incidence.

Results: We identified 36 studies of which 2 were metaanalyses, 5 were randomized controlled trials, 9 were prospective studies, and 20 were retrospective studies. When anakinra was compared with control, 2 studies reported an increased risk of infection, and 3 studies reported a similar or decreased incidence of infection. Canakinumab had a lower associated incidence of infection compared with placebo in one study. When sarilumab was compared with placebo, one study reported an increased risk of infection. Nine studies comparing tocilizumab with placebo reported decreased or no dif- 
ference in infection risk (odds ratio [OR] for the studies ranged from 0.39-1.21). Fourteen studies comparing tocilizumab with placebo reported an increased risk of infection, ranging from $9.1 \%$ to $63.0 \%$ (OR for the studies ranged from 1.85-5.04). Infection most commonly presented as bacteremia. Of the 6 studies comparing tocilizumab and corticosteroid use with placebo, 4 reported a nonsignificant increase toward corticosteroids being associated with bacterial infections (OR ranged from 2.76-3.8), and 2 studies reported no increased association with a higher infection risk.

Conclusions: Our literature review showed mixed results with variable significance for the association of IL-6 inhibitors with risk of infections in patients with COVID-19.

Keywords: Corticosteroid, COVID-19, cytokine storm, infection, interleukin inhibitor.

\section{INTRODUCTION}

CARS-CoV-2 was first identified in late 2019 as a cause of acute respiratory illness in Wuhan, China [1]. In February 2020, the World Health Organization (WHO) termed the viral disease COVID-19 for coronavirus disease 2019 [2]. Since then, more than 184 million confirmed cases have been identified globally (July 8, 2021). Patients are at increased risk of spreading the infection in the early stages of illness, even before symptom onset, which has led to substantial morbidity and mortality. Patients admitted to the intensive care unit (ICU) have been reported to have an especially high mortality rate, prompting trials of several investigational agents as treatments and changing practice patterns as evidence accumulates in real time [3]. Key management strategies so far include supportive care, therapy directed at viral replication, and immune-modulatory therapy.

Interleukin (IL) inhibitors and antagonists are biologic agents now being used off label to treat COVID-19 based on their use in treating other diseases. However, they have been associated with adverse events. When tocilizumab was used for rheumatologic conditions, 6 to 10 serious infections per 100 patient-years were reported [4]. The rates of infection per patient-year were similar when siltuximab was used to treat patients with non-Hodgkin lymphoma (5.2), Castleman disease (1.9), or multiple myeloma (1.8) [5]. Serious infections were also noted in a large, multicenter placebo-controlled trial of patients with rheumatoid arthritis treated with anakinra (2.1\% vs $0.4 \%)$ [6].

$\overline{\text { Corresponding author }}$

Thoyaja Koritala

E-mail: koritala.thoyaja@mayo.edu
We were unable to find any data about the infection incidence associated with sarilumab in treatment of non-COVID-19 disease. The risk of secondary infection for patients with COVID-19 after treatment with IL inhibitors is not well described. Therefore, for this review, we aimed to determine whether an increase in infection risk occurred for hospitalized patients with COVID-19 treated with IL inhibitors, with or without the use of corticosteroids.

\section{METHODS}

A literature search was conducted using the following evidence-based medicine reviews: Cochrane Central Register of Controlled Trials March 2021; Cochrane Database of Systematic Reviews 2005 to April 28, 2021; Embase 1974 to April 28, 2021; Ovid Medline; and Epub Ahead of Print, In-Process, In-Data-Review \& Other NonIndexed Citations, Daily and Versions 1946 to April 28, 2021. All relevant articles were identified by using the following search terms: coronavirus disease 2019, COVID-19, SARS-coronavirus-2, infections, interleukins, inpatients, adults, and incidence. The literature search was limited to English language and studies involving humans. We excluded review articles, case reports, opinion articles, letters, abstracts, and brief reports. We also reviewed references of the identified articles to find additional relevant studies. All studies were imported into Endnote X9 (Clarivate Analytics), and duplicate articles were identified and removed (Figure 1).

\section{Pathophysiology}

SARS-CoV-2 enters the human body via interaction with angiotensin-converting enzyme 2 (ACE2 ) receptors and releases its RNA inside epithelial 
cells, where it replicates. From the nasal epithelial cells, SARS-CoV-2 spreads to the alveoli of the lungs [7]. Endothelial injury is a key consequential pathologic event, leading to multiorgan failure. Compromise of vascular integrity of the alveoli results in pulmonary edema, disseminated intravascular coagulation, pulmonary ischemia, hypoxic respiratory failure, and progressive lung damage [8]. After SARS-CoV-2 invades epithelial cells by binding with ACE-2 receptors, localized inflammation, endothelial activation, tissue damage, and dysregulated cytokine release begins.

Cytokine storm starts with secretion of vascular endothelial growth factor, monocyte chemoattractant protein-1, IL-1, IL-2, IL-6, IL-8, IL-10, tumor necrosis factor- $\alpha$, interferon- $\gamma$, and reduced E-cadherin expression on epithelial cells [9-11]. High levels of cytokines potentiate inflammation, epithelial and endothelial cell dysfunction, vasodilation of the capillary bed resulting in acute respiratory distress syndrome (ARDS), multiorgan failure, and death. Increased levels of cytokines and chemokines are associated with severity of COVID-19. Therapeutic interventions to normal- ize the vasculature include use of anti-inflammatory drugs, ACE inhibitors, and anti-cytokine drugs [12]. Cytokine inhibitors are being used to contain the cytokine storm effectively [12].

\section{IL inhibitor use in the treatment of COVID-19}

The IL inhibitors tocilizumab, sarilumab, siltuximab, canakinumab, and anakinra have been used to treat COVID-19. Tocilizumab and sarilumab are classified as IL-6 receptor antagonists, and siltuximab is a direct IL-6 inhibitor. Anakinra and canakinumab are IL-1 receptor antagonists. Care should be taken to avoid beginning immunosuppressive biologic therapy for patients with active infection, and therapy should be discontinued if a serious infection develops with its use. Recrudescence of latent tuberculosis or other opportunistic infections is a known adverse effect with immunosuppressive biologic drugs [13].

From our search, we identified 36 studies describing the use of IL inhibitors for patients with COVID-19: 2 meta-analyses, 5 randomized controlled trials, 9 prospective studies, and 20 retrospective studies. When more than $1 \mathrm{IL}$ inhibitor was

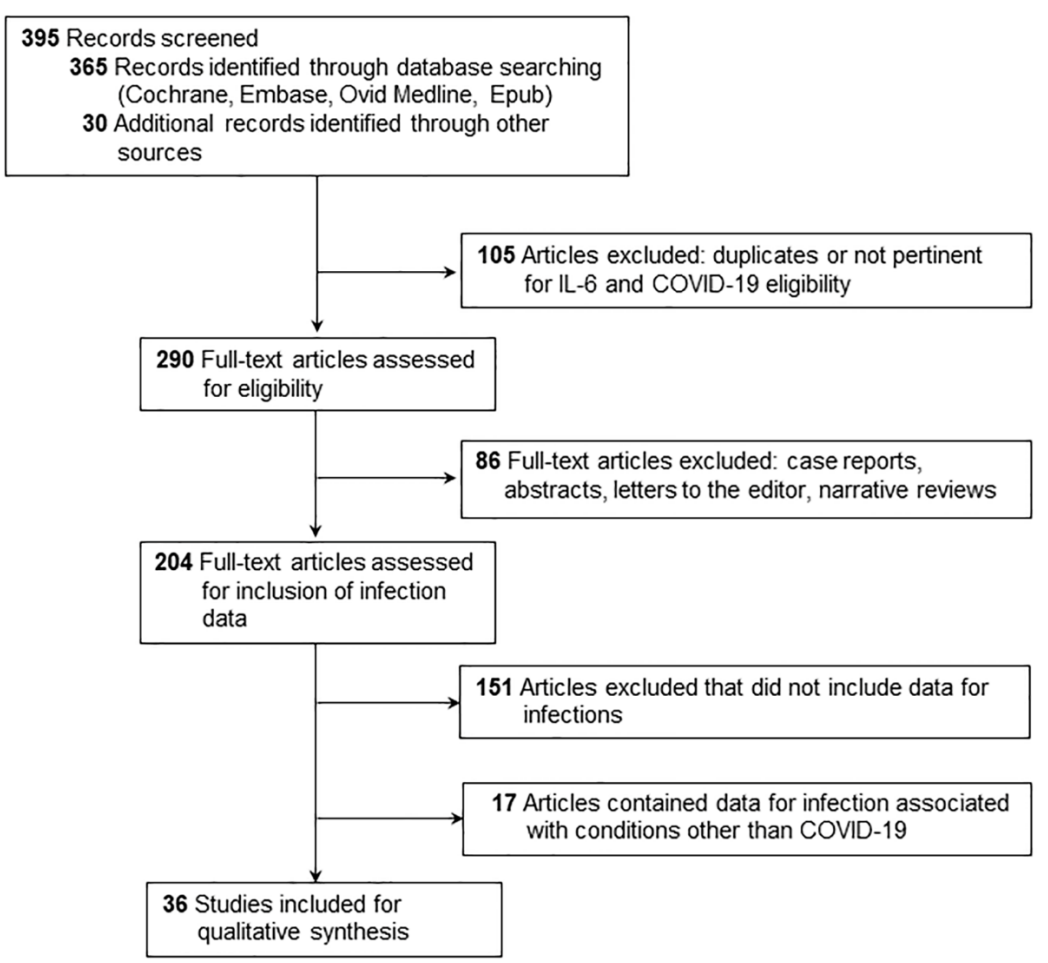

Figure 1 - Flow diagram showing the strategy for articles included in the review. 
included in a study, it was described in the outcome section where it was most appropriate. We also included the relevant studies that described infections associated with IL inhibitor use in conditions other than COVID-19.

\section{Tocilizumab}

Tocilizumab is an IL-6 receptor antagonist used as a disease-modifying antirheumatic drug. By acting as an inhibitor to IL-6 receptors, tocilizumab reduces cytokines and acute-phase reactant production and pauses or calms the immunologic response, thereby making the diagnosis of secondary infection difficult [14]. Labeled indications are rheumatoid arthritis, giant cell arteritis, systemic and polyarticular juvenile idiopathic arthritis, and systemic sclerosis-associated interstitial lung disease. Severe and potentially lethal cytokine-release syndrome and COVID-19 infection are offlabel indications of tocilizumab.

The Infectious Diseases Society of America suggested adding tocilizumab to the corticosteroid regimen for hospitalized patients with severe or critical COVID-19 and increased inflammatory markers [15]. Patients with an oxygen saturation less than $92 \%$ on room air and a C-reactive protein level greater than $75 \mathrm{mg} / \mathrm{L}$ who required high-flow oxygen and noninvasive or invasive mechanical ventilation in the past 24 hours were considered to have severe or critical COVID-19 infection.

\section{Clinical data}

Most recently, the WHO Rapid Evidence Appraisal for COVID-19 Therapies (REACT) working group conducted a prospective meta-analysis of 10,930 patients participating in 27 clinical trials. The meta-analysis identified a lower 28-day, allcause mortality of $22 \%$ for patients treated with IL-6 receptor antagonists compared with $25 \%$ in a placebo group (odds ratio [OR], 0.83 [95\% CI, 0.74-0.92]; $p<.001$ for tocilizumab and OR, 1.08 [95\% CI, 0.86-1.36]; $p=.52$ for sarilumab) [16]. Results from 2 large prospective randomized controlled trials showed similar mortality benefits of tocilizumab: the Randomized Evaluation of COVID-19 Therapy (RECOVERY) trial and the Randomized, Embedded, Multifactorial Adaptive Platform Trial for Community-Acquired Pneumonia (REMAP-CAP) trial with tocilizumab $[17,18]$. The REMAP-CAP trial showed a mortality benefit from tocilizumab (27\%) vs a control group (36\%) (adjusted OR for survival, 1.64 [95\% CI, 1.142.35]). Sarilumab was also shown to improve survival in the REMAP-CAP trial $[18,19]$. Similarly, the RECOVERY trial showed a mortality benefit from tocilizumab: $29 \%$ for the tocilizumab group vs 33\% for the control group (OR, 0.86 [95\% CI, 0.77-0.96]) [17].

A meta-analysis of 8 randomized controlled trials involving 6,311 hospitalized patients with COVID-19 given tocilizumab suggested benefits for mortality and the need for mechanical ventilation when the tocilizumab group was compared with placebo or standard therapy [20]. However, several other trials did not identify a mortality benefit or other clear clinical benefit with tocilizumab or sarilumab [21-27].

Secondary infection was defined as any positive culture for bacterial or fungal organisms from blood or other sterile sites [28]. In patients with critical disease and a poor prognosis, more bacteremia and secondary infections were noted with the use of an IL inhibitor [29-33]. It is unclear whether the increase in infection risk was due to the advanced stage of the disease and underlying comorbid conditions potentiated by the use of IL inhibitors or by the IL inhibitors themselves.

The most recent evidence of secondary infection occurring by 28 days was published in a WHOconducted meta-analysis and showed increased risk of infection for patients treated with IL-6 antagonists compared with patients treated with placebo or standard care [16]. A meta-analysis conducted by Han et al. showed increased risk of infection with the use of IL-6 antagonists in patients with critical disease [34]. No such increase was noted for severe disease. No statistical significance was shown for the overall risk of secondary infection with the use of IL-6 antagonists in patients with critical or severe COVID-19 disease. Two randomized controlled trials conducted by Rosas et al. and Stone et al. showed fewer infections in a group given tocilizumab than in a control group [22, 24]. Several prospective and retrospective studies showed a non-significant increased risk of infection with the use of tocilizumab in critically ill patients with COVID-19 with or without the need for mechanical ventilation [34-38]. The most common presentation was bloodstream infection followed by bacterial pneumonia. Gram-positive bacteremia occurred more 
often than Gram-negative bacteremia, fungemia, or viremia. Staphylococcus aureus was the causative organism in about $50 \%$ of patients with bacterial pneumonia [29]. When used in conjunction with tocilizumab, corticosteroids were associated with a nonsignificant increase in infection in critically ill patients with COVID-19 [39].

In larger retrospective studies conducted by Guaraldi et al. and Gupta et al., secondary infections occurred more often in the tocilizumab group than the placebo group $(p<.001)[32,33]$. Pettit et al. reported more late-onset infections (more than 48 hours) in a tocilizumab group than a placebo group ( $p=.01)$ [40]. Rodriguez-Bano et al. reported that the use of corticosteroids in a pulsed dose, in an intermittent high dose, or in combination with tocilizumab did not increase the risk of secondary infections [36]. In a large retrospective study of 1,565 hospitalized patients with COVID-19, Kumar et al. reported significantly more health care-associated infections after treatment with tocilizumab $(p<.001)$ and corticosteroids $(p=.007)$ [41]. Nine studies comparing tocilizumab with controls showed decreased or no difference in risk of secondary infections, and 15 studies reported an increased risk of infections ranging from $9.1 \%$ to $63.0 \%$, with the most common infection presentation reported as bacteremia followed by pneumonia and urinary tract infection $[16,22$, $24,28,29,31-41,42-49]$. Of 6 studies comparing tocilizumab and corticosteroid use with a control group, 4 studies showed corticosteroid treatment to be associated with a nonsignificant increase in bacterial infections, and 2 studies showed no increased risk of infection [30, 36, 39, 41, 47].

Data for infection risk were similar when tocilizumab was used to treat patients with rheumatologic conditions along with methotrexate. In a multicenter, randomized placebo-controlled trial of 499 patients with rheumatoid arthritis, the rate of serious infections with high-dose tocilizumab $(8 \mathrm{mg} / \mathrm{kg})$, low-dose tocilizumab $(4 \mathrm{mg} / \mathrm{kg})$, and placebo were $4.6 \%, 1.8 \%$, and $3.1 \%$ respectively [4]. Thus, serious infections occurred more often after patients were treated with high-dose tocilizumab for COVID-19 or other conditions.

\section{Sarilumab}

IL-6 is produced by inflammatory stimuli. Sarilumab is an IL-6 receptor antagonist that is a monoclonal antibody and disease-modifying antirheumatic drug that is indicated by label to treat rheumatoid arthritis [50]. The most common serious infections after treatment with sarilumab are pneumonia, urinary tract infection, and cellulitis; opportunistic infections like tuberculosis, candidiasis, and pneumocystis have also been reported [51].

Della-Torre et al. conducted an open-label observational prospective study of 56 patients with COVID-19 and severe pneumonia, which showed sarilumab to have an associated infection rate of $21 \%$ compared with an infection rate of $18 \%$ for control patients [52]. In an observational study of 255 patients with COVID-19 and pneumonia, $34(13.3 \%)$ patients who received sarilumab or tocilizumab had secondary bacterial infections more than 48 hours after receiving the IL- 6 inhibitor [53]. Four (11.8\%) of these patients eventually died. Secondary infections occurred with an OR of 0.77 (95\% CI, 0.39-1.58) and were not related to a higher risk of mortality (OR, 1.09 [95\% CI, 0.363.38]) or a lower rate of discharge (OR, $0.76[95 \%$ CI, 0.29-1.99]).

\section{Siltuximab}

Siltuximab is an IL-6 inhibitor, chimeric monoclonal antibody, and an antineoplastic agent. Siltuximab binds with IL-6 and stops it from attaching to IL-6 receptors. The labeled indication for siltuximab is multicentric HIV and human herpesvirus 8-negative Castleman disease [54]. In a phase I study that enrolled 67 patients with non-Hodgkin lymphoma, Castleman disease, or multiple myeloma, patients received siltuximab at 3,6, 9, or $12 \mathrm{mg} / \mathrm{kg}$ weekly, every 2 to 3 weeks [5]. Of the patients, $44(66 \%)$ had all-grade adverse infection events. Per patient-year, the infection event rate was 5.2 for non-Hodgkin lymphoma, 1.9 for Castleman disease, and 1.8 for multiple myeloma. The all-grade infection event rate per patient-year was 2.1 for all treated patients. The most common infections were upper respiratory tract $(39 \%)$; urinary tract $(16 \%)$; ear $(6 \%)$, nose $(7 \%)$, and throat $(6 \%$ $12 \%)$; and cellulitis (9\%). We did not identify any studies about the use of siltuximab and its association with infection for patients with COVID-19.

\section{Anakinra}

Anakinra is an IL-1 receptor antagonist used as a disease-modifying antirheumatic drug. Any inflammation incites IL-1 production and varied immunologic reactions. Anakinra halts the 
immunologic response. Labeled indications for anakinra are rheumatoid arthritis, deficiency of IL-1 receptor antagonists, and neonatal onsetmultisystem inflammatory disease [55]. Off-label adult indications are acute gout flares, recurrent pericarditis, and familial Mediterranean fever. A $39 \%$ overall infection incidence was reported for anakinra use, mostly manifesting as pneumonia, bone and/or joint infections, and cellulitis for patients with rheumatoid arthritis [56]. In a large, multicenter, international placebo-controlled trial, serious infections occurred at a higher rate in the anakinra group for patients with rheumatoid arthritis $(2.1 \%$ vs $0.4 \%)$ [6]. The study enrolled 1,414 patients who were randomly assigned to a daily, 100-mg injection of anakinra or placebo administered subcutaneously.

Anakinra has more recently been used to treat COVID-19. In a prospective, open-label, interventional study of 69 hospitalized patients with severe COVID-19 pneumonia, Balkhair et al. showed that anakinra decreased bloodstream infections from $18 \%$ for control patients to $11 \%$ for patients treated with anakinra $(p=.46)$ [57]. Results of a prospective cohort study of 60 intubated patients with COVID-19 pneumonia showed that 7 patients $(33 \%)$ who received anakinra developed a secondary infection in the first 28 days compared with 9 patients $(23 \%)$ in the control group $(p=.54)$ [58]. Similarly, in a retrospective cohort study of 52 patients with COVID-19 with moderate to severe ARDS who were treated with noninvasive ventilation outside of an ICU, bacteremia (Staphylococcus epidermidis) developed in 4 of 29 patients (14\%) treated with high-dose anakinra (5 $\mathrm{mg} / \mathrm{kg}$ intravenous twice daily) compared with 2 of 16 patients (13\%) not treated with anakinra. Not enough data were available for 7 patients who received low-dose subcutaneous anakinra (100 mg twice daily) to make meaningful conclusions [59].

The SAVE-MORE study (suPAR-Guided Anakinra Treatment for Validation of the Risk and Early Management of Severe Respiratory Failure by COVID-19) is a crucial phase 3, double-blind, randomized, controlled multicenter trial that assessed the effectiveness and safety of early administration of anakinra therapy for hospitalized patients with moderate or severe COVID-19. Of 594 patients in an intention-to-treat cohort, infections were identified less in the anakinra group than in the placebo group (34/405 [8.4\%] vs $30 / 189$ [15.9\%]; $p=.01$ ). Commonly observed infections were ventilator-associated pneumonia (9/405 [2.2\%] vs $15 / 189$ [7.9\%]; $p=.003)$; septic shock and multiple organ dysfunction (6/405 [1.5\%] vs $7 / 189$ [3.7\%]; $p=.13)$ and bacteremia $(12 / 405[3.0 \%]$ vs $6 / 189$ [3.2\%]; $p=1.00)$ in the anakinra and placebo groups, respectively. A lower incidence of infections, which reached statistical significance, was reported for patients treated with anakinra [60].

CORIMUNO-19 (Cohort Multiple Randomized Controlled Trials Open-Label of Immune Modulatory Drugs and Other Treatments in COVID-19 Patients) is an open-label, multicenter, randomized controlled trial that was conducted in France and enrolled 114 patients with mild to moderate COVID-19 pneumonia. The CORIMUNO-19 collaborative group reported a higher incidence of bacterial (10/59) and fungal sepsis (1/59) in the anakinra group vs the usual-care group, which had only bacterial sepsis $(4 / 55 ; p=.099)$ [61].

For studies comparing anakinra with placebo, 2 showed an increased risk of infections, and 3 showed a similar, decreased incidence of infections [57-61]. Thus, a nonsignificant association of anakinra and increased infection was shown for patients with COVID-19 and with other nonCOVID-19 conditions, such as rheumatologic disease.

\section{Canakinumab}

Canakinumab, like anakinra, is an IL-1 antagonist that was compared with placebo in a randomized, double-blind, placebo-controlled multicenter trial that enrolled 448 patients with COVID-19 pneumonia. The results favored canakinumab over placebo for pneumonia $(6 / 225$ [2.7\%] vs $7 / 223$ [3.1\%]), infections $(23 / 225$ [10.2\%] vs $43 / 223$ [19.3\%]), and serious infections $(11 / 225[4.9 \%]$ vs $21 / 223[9.4 \%])[62]$.

\section{Limitations}

We excluded case reports, abstracts, letters to the editor, and narrative reviews from our study, which may have contributed to the further understanding of the association of infection with IL inhibitor use for patients with COVID-19. Although it would have been ideal to include data for both efficacy and infection incidence with use of IL inhibitors in treating COVID-19, given the size and 
complexity of the presentation, we only included infection risk in this review.

\section{CONCLUSIONS}

We found mixed results and varied significance for an association between IL inhibitors and increased risk of infection for patients with COVID-19. Well-designed, large, prospective randomized controlled trials and/or meta-analyses, as evidence accumulates, will be required to delineate any true association.

\section{Conflict of interest}

None.

\section{Funding}

None.

\section{Acknowledgment}

Marianne Mallia, Mayo Clinic, substantively edited the manuscript. The Scientific Publications staff at Mayo Clinic provided proofreading and administrative and clerical support.

\section{Authors contribution}

T.K., V.P., R.T., A.L., O.M.A., R.K., and S.S. were involved with idea origination, literature review, writing, and review of the manuscript. A.R., A.A., S.A., R.A., K.D., N.K.J., V.B., A.T., S.Z., S.A.K., and J.P.D.G. were involved in writing and review of the manuscript. T.K. served as the lead author on the manuscript and was responsible for finalizing the reviewed literature, compiling the penned data of multiple authors, rewriting the manuscript and including other authors' comments, and returning the manuscript drafts to the other authors for their subsequent reviews. All authors approved the final version.

\section{REFERENCES}

[1] Shah A, Kashyap R, Tosh P, Sampathkumar P, O'Horo JC. Guide to understandingthe 2019 novel coronavirus. Mayo Clin Proc. 2020; 95 (4), 646-52.

[2] World Health Organization. WHO Director-General's remarks at the media briefing on 2019-nCoV on 11 February 2020. Geneva: World Health Organization; 2020. Available at: https://www.who.int/directorgeneral/speeches / detail/who-director-general-s-remarks-at-the-media-briefing-on-2019-ncov-on-11-february-2020. [Accessed 2021-Aug 5].
[3] Domecq JP, Lal A, Sheldrick CR, et al. Outcomes of patients with coronavirus disease 2019 receiving organ support therapies: The International Viral Infection and Respiratory Illness Universal Study Registry. Crit Care Med. 2021; 49 (3), 437-48.

[4] Emery P, Keystone E, Tony HP, et al. IL-6 receptor inhibition with tocilizumab improves treatment outcomes in patients with rheumatoid arthritis refractory to anti-tumour necrosis factor biologicals: results from a 24-week multicentre randomised placebo-controlled trial. Ann Rheum Dis. 2008; 67 (11), 1516-23.

[5] Kurzrock R, Voorhees PM, Casper C, et al. A phase I, open-label study of siltuximab, an anti-IL-6 monoclonal antibody, in patients with B-cell non-Hodgkin lymphoma, multiple myeloma, or Castleman disease. Clin Cancer Res. 2013; 19 (13), 3659-70.

[6] Fleischmann RM, Schechtman J, Bennett R, et al. Anakinra, a recombinant human interleukin-1 receptor antagonist (r-metHuIL-1ra), in patients with rheumatoid arthritis: a large, international, multicenter, placebo-controlled trial. Arthritis Rheum. 2003; 48 (4), 927-34. [7] Mason RJ. Pathogenesis of COVID-19 from a cell biology perspective. Eur Respir J. 2020; 55 (4).

[8] Teuwen LA, Geldhof V, Pasut A, Carmeliet P. Author correction: COVID-19: the vasculature unleashed. Nat Rev Immunol. 2020; 20 (7), 448.

[9] Coperchini F, Chiovato L, Croce L, Magri F, Rotondi M. The cytokine storm in COVID-19: an overview of the involvement of the chemokine/chemokine-receptor system. Cytokine Growth Factor Rev. 2020; 53, 25-32. [10] Saha A, Sharma AR, Bhattacharya M, Sharma G, Lee SS, Chakraborty C. Tocilizumab: a therapeutic option for the treatment of cytokine Storm Syndrome in COVID-19. Arch Med Res. 2020; 51 (6), 595-7.

[11] Costela-Ruiz VJ, Illescas-Montes R, Puerta-Puerta JM, Ruiz C, Melguizo-Rodriguez L. SARS-CoV-2 infection: the role of cytokines in COVID-19 disease. Cytokine Growth Factor Rev. 2020; 54, 62-75.

[12] Pober JS, Sessa WC. Evolving functions of endothelial cells in inflammation. Nat Rev Immunol. 2007; 7 (10), 803-15.

[13] Troskot B, Simunic M. [Side effects and contraindications for biological therapy in inflammatory bowel disease]. Acta Med Croatica. 2013; 67 (2), 131-43.

[14] Tirupathi R, Bharathidasan K, Areti S, Kaur J, Salim S, Al-Tawfiq JA. The shortcomings of tocilizumab in COVID-19. Infez Med. 2020; 28 (4), 465-8.

[15] Bhimraj A, Morgan RL, Shumaker AH, et al. Infectious Diseases Society of America Guidelines on the treatment and management of patients with $\mathrm{CO}-$ VID-19. Arlington (VA): Infectious Diseases Society of America; 2021. Available at: https://www.idsociety. org/practice-guideline/covid-19-guideline-treatmentand-management/. [Accessed 2021 Aug 5].

[16] WHO. Rapid Evidence Appraisal for COVID-19 Therapies Working Group, Shankar-Hari M, Vale CL, 
et al. Association between administration of IL-6 antagonists and mortality among patients hospitalized for COVID-19: a meta-analysis. JAMA. 2021.

[17] Recovery Collaborative Group. Tocilizumab in patients admitted to hospital with COVID-19 (RECOVERY): a randomised, controlled, open-label, platform trial. Lancet. 2021; 397 (10285), 1637-45.

[18] Remap-Cap Investigators, Gordon AC, Mouncey $\mathrm{PR}$, et al. Interleukin-6 receptor antagonists in critically ill patients with COVID-19. N Engl J Med. 2021; 384 (16), 1491-502.

[19] The REMAP-CAP Investigators, Derde LPG. Effectiveness of tocilizumab, sarilumab, and anakinra for critically ill patients with COVID-19: the REMAP-CAP COVID-19 immune modulation therapy domain randomized clinical trial. BMJ Yale: Cold Spring Harbor Laboratory; 2021. Available at: https://www.medrxiv. org/content/10.1101/2021.06.18.21259133v2. [Accessed 2021 Oct 27].

[20] Selvaraj V, Khan MS, Bavishi C, et al. Tocilizumab in hospitalized patients with COVID-19: a meta-analysis of randomized controlled trials. Lung. 2021; 199 (3), 239-48.

[21] Sanofi. Sanofi and Regeneron provide update on Kevzara ${ }^{\circledR}$ (sarilumab) Phase 3 US trial in COVID-19 patients. Sanofi; 2020. Available at: https:// www.san ofi.com/en/media-room/press-releases/2020/2020-07-02-22-30-00. [Accessed 2021 Aug 5].

[22] Rosas IO, Brau N, Waters M, et al. Tocilizumab in hospitalized patients with severe COVID-19 pneumonia. N Engl J Med. 2021; 384 (16), 1503-16.

[23] Parodi E, O'Donnell C. Roche rheumatoid arthritis drug fails to help COVID-19 patients in Italian study. London: Reuters; 2020. Available at: https://www. reuters.com/article/us-health-coronavirus-rochehldg/roche-rheumatoid-arthritis-drug-fails-to-help-covid-19-patients-in-italian-study-idUSKBN23O3GG. [Accessed 2020 Aug 5].

[24] Stone JH, Frigault MJ, Serling-Boyd NJ, et al. Efficacy of tocilizumab in patients hospitalized with $\mathrm{CO}-$ VID-19. N Engl J Med. 2020; 383 (24), 2333-44.

[25] Hermine O, Mariette X, Tharaux PL, et al. Effect of tocilizumab vs usual care in adults hospitalized with COVID-19 and moderate or severe pneumonia: a randomized clinical trial. JAMA Intern Med. 2021; 181 (1), 32-40.

[26[ Salvarani C, Dolci G, Massari M, et al. Effect of tocilizumab vs standard care on clinical worsening in patients hospitalized with COVID-19 pneumonia: a randomized clinical trial. JAMA Intern Med. 2021; 181 (1), 24-31.

[27] Salama C, Han J, Yau L, et al. Tocilizumab in patients hospitalized with COVID-19 pneumonia. N Engl J Med. 2021; 384 (1), 20-30.

[28] Mehta M, Purpura LJ, McConville TH, et al. What about tocilizumab? A retrospective study from a NYC hospital during the COVID-19 outbreak. PLoS One. 2021; 16 (4), e0249349.

[29] Somers EC, Eschenauer GA, Troost JP, et al. Tocilizumab for treatment of mechanically ventilated patients with COVID-19. Clin Infect Dis. 2021; 73 (2), e445-e54.

[30] Buetti N, Ruckly S, de Montmollin E, et al. COVID-19 increased the risk of ICU-acquired bloodstream infections: a case-cohort study from the multicentric OUTCOMEREA network. Intensive Care Med. 2021; 47 (2), 180-7.

[31] Biran N, Ip A, Ahn J, et al. Tocilizumab among patients with COVID-19 in the intensive care unit: a multicentre observational study. Lancet Rheumatol. 2020; 2 (10), e603-e12.

[32] Guaraldi G, Meschiari M, Cozzi-Lepri A, et al. Tocilizumab in patients with severe COVID-19: a retrospective cohort study. Lancet Rheumatol. 2020; 2 (8), e474-e84.

[33] Gupta S, Wang W, Hayek SS, et al. Association between early treatment with tocilizumab and mortality among critically ill patients with COVID-19. JAMA Intern Med. 2021; 181 (1), 41-51.

[34] Han Q, Guo M, Zheng Y, et al. Current evidence of interleukin-6 signaling inhibitors in patients with $\mathrm{CO}-$ VID-19: a systematic review and meta-analysis. Front Pharmacol. 2020; 11, 615972.

[35] Roumier M, Paule R, Vallee A, et al. Tocilizumab for severe worsening COVID-19 pneumonia: a propensity score analysis. J Clin Immunol. 2021; 41 (2), 303-14.

[36] Rodriguez-Bano J, Pachon J, Carratala J, et al. Treatment with tocilizumab or corticosteroids for COVID-19 patients with hyperinflammatory state: a multicentre cohort study (SAM-COVID-19). Clin Microbiol Infect. 2021; 27 (2), 244-52.

[37] Vazquez Guillamet MC, Kulkarni HS, Montes K, et al. Interleukin-6 trajectory and secondary infections in mechanically ventilated patients with coronavirus disease 2019 Acute Respiratory Distress Syndrome treated with Interleukin-6 receptor bBlocker. Crit Care Explor. 2021; 3 (2), e0343.

[38] Campochiaro C, Della-Torre E, Cavalli G, et al. Efficacy and safety of tocilizumab in severe COVID-19 patients: a single-centre retrospective cohort study. Eur J Intern Med. 2020; 76, 43-9.

[39] Kimmig LM, Wu D, Gold M, et al. IL-6 inhibition in critically ill COVID-19 patients is associated with increased secondary infections. Front Med (Lausanne). 2020; 7, 583897.

[40] Pettit NN, Nguyen CT, Mutlu GM, et al. Late onset infectious complications and safety of tocilizumab in the management of COVID-19. J Med Virol. 2021; 93 (3), 1459-64.

[41] Kumar G, Adams A, Hererra M, et al. Predictors and outcomes of healthcare-associated infections in COVID-19 patients. Int J Infect Dis. 2021; 104, 287-92. 
[42] Canziani LM, Trovati S, Brunetta E, et al. Interleukin-6 receptor blocking with intravenous tocilizumab in COVID-19 severe acute respiratory distress syndrome: a retrospective case-control survival analysis of 128 patients. J Autoimmun. 2020; 114, 102511.

[43] Eimer J, Vesterbacka J, Svensson AK, et al. Tocilizumab shortens time on mechanical ventilation and length of hospital stay in patients with severe COVID-19: a retrospective cohort study. J Intern Med. 2021; 289 (3), 434-6. [44] Fisher MJ, Marcos Raymundo LA, Monteforte M, Taub EM, Go R. Tocilizumab in the treatment of critical COVID-19 pneumonia: a retrospective cohort study of mechanically ventilated patients. Int J Infect Dis. 2021; 103, 536-9.

[45] Rojas-Marte G, Khalid M, Mukhtar O, et al. Outcomes in patients with severe COVID-19 disease treated with tocilizumab: a case-controlled study. QJM. 2020; 113 (8), 546-50.

[46] Huang E, Isonaka S, Yang H, Salce E, Rosales E, Jordan SC. Tocilizumab treatment in critically ill patients with COVID-19: a retrospective observational study. Int J Infect Dis. 2021; 105, 245-51.

[47] Balena F, Bavaro DF, Fabrizio C, et al. Tocilizumab and corticosteroids for COVID-19 treatment in elderly patients. J Gerontol Geriatr. 2020; 68 (4).

[48] Maeda T, Obata R, Rizk DD, Kuno T. The association of interleukin-6 value, interleukin inhibitors, and outcomes of patients with COVID-19 in New York City. J Med Virol. 2021; 93 (1), 463-71.

[49] Chamorro-de-Vega E, Rodriguez-Gonzalez CG, Manrique-Rodriguez S, et al. Clinical course of severe patients with COVID-19 treated with tocilizumab: report from a cohort study in Spain. Expert Rev Clin Pharmacol. 2021; 14 (2), 249-60.

[50] Science Direct. Amsterdam (Netherlands): Elsevier. Available at: https://www.sciencedirect.com/topics/ medicine-and-dentistry/sarilumab. [Accessed 2021 Aug 19]. [51] UpToDate. Sarilumab: drug information. 2021. Available at: https://www.uptodate.com/contents / sarilumab-drug-information?search=sarilumab\&sou rce=panel_search_result\&selectedTitle $=1 \sim 21 \&$ usage_ type $=$ panel\&kp_tab=drug_general\&display_ rank=1\#F50136516. [Accessed 2021 Aug 19].

[52] Della-Torre E, Campochiaro C, Cavalli G, et al. Interleukin-6 blockade with sarilumab in severe $\mathrm{CO}$ VID-19 pneumonia with systemic hyperinflammation: an open-label cohort study. Ann Rheum Dis. 2020; 79 (10), 1277-85.
[53] Sinha P, Mostaghim A, Bielick CG, et al. Early administration of interleukin-6 inhibitors for patients with severe COVID-19 disease is associated with decreased intubation, reduced mortality, and increased discharge. Int J Infect Dis. 2020; 99, 28-33.

[54]UpToDate.Siltuximab: drug information. UpToDate; 2020. Available at: https: / / www.uptodate.com/contents / siltuximab-drug-information?search=siltuximab\&sou rce=panel_search_result\&selectedTitle=1 11\&usage_ type $=$ panel\&kp_tab=drug_general\&display_rank=1. [Accessed 2021 Aug 5].

[55] UpToDate. Anakinra: drug information. UpToDate; 2020. Available at: https://www.uptodate.com/ contents / anakinra-drug-information? search=anakin ra\&source=panel_search_result\&selectedTitle $=1 \sim 111$ \&usage_type $=$ panel\&kp_tab=drug_general\&display_ rank=1. [Accessed 2021 Aug 5].

[56] Fleischmann RM, Tesser J, Schiff MH, et al. Safety of extended treatment with anakinra in patients with rheumatoid arthritis. Ann Rheum Dis. 2006; 65 (8), 1006-12.

[57] Balkhair A, Al-Zakwani I, Al Busaidi M, et al. Anakinra in hospitalized patients with severe COVID-19 pneumonia requiring oxygen therapy: results of a prospective, open-label, interventional study. Int J Infect Dis. 2021; 103, 288-96.

[58] Kooistra EJ, Waalders NJB, Grondman I, et al. Anakinra treatment in critically ill COVID-19 patients: a prospective cohort study. Crit Care. 2020; 24 (1), 688.

[59] Cavalli G, De Luca G, Campochiaro C, et al. Interleukin-1 blockade with high-dose anakinra in patients with COVID-19, acute respiratory distress syndrome, and hyperinflammation: a retrospective cohort study. Lancet Rheumatol. 2020; 2 (6), e325-e31.

[60] Kyriazopoulou E, Poulakou G, Milionis H, et al. Early treatment of COVID-19 with anakinra guided by soluble urokinase plasminogen receptor plasma levels: a double-blind, randomized controlled phase 3 trial. Nat Med. 2021; 27 (10), 1752-60.

[61] Corimuno-Collaborative group. Effect of anakinra versus usual care in adults in hospital with COVID-19 and mild-to-moderate pneumonia (CORIMUNOANA-1): a randomised controlled trial. Lancet Respir Med. 2021; 9 (3), 295-304.

[62] Caricchio R, Abbate A, Gordeev I, et al. Effect of canakinumab vs placebo on survival without invasive mechanical ventilation in patients hospitalized with severe COVID-19: a randomized clinical trial. JAMA. $2021 ; 326$ (3), 230-9. 